IFT-P.001/2008

$\mathrm{UCLA} / 08 / \mathrm{TEP} / 1$

\title{
Pure Spinor Superspace Identities for Massless Four-point Kinematic Factors
}

\author{
Carlos R. Mafral \\ Instituto de Física Teórica, State University of São Paulo \\ Rua Pamplona 145, 01405-900, São Paulo, SP, Brasil \\ Department of Physics and Astronomy \\ University of California, Los Angeles, CA 90095
}

\begin{abstract}
Using the pure spinor formalism we prove identities which relate the tree-level, oneloop and two-loop kinematic factors for massless four-point amplitudes. From these identities it follows that the complete supersymmetric one- and two-loop amplitudes are immediately known once the tree-level kinematic factor is evaluated. In particular, the two-loop equivalence with the RNS formalism (up to an overall coefficient) is obtained as a corollary.
\end{abstract}

January 2008

1 email: crmafra@ift.unesp.br, crmafra@physics.ucla.edu 


\section{Introduction}

Despite numerous successes in the non-perturbative front, perturbative studies of superstring theory remain an important subject of its own. For example, by computing graviton scattering amplitudes one can catch a glimpse of quantum gravity at work in its deepest realms, from which one can derive superstring modifications of Einstein's equations [1].

Since the birth of the pure spinor formalism [2], the task of computing superstring scattering amplitudes has been made easier [3] [4] [5]. Its main advantage over the standard Ramond-Neveu-Schwarz (RNS) and Green-Schwarz (GS) formulations is due to covariant quantization being possible while having manifest space-time supersymmetry. These properties help simplify the required computations, which can be done efficiently. However, until its origins are fully explained [6] [7] [8] and a general proof of equivalence with the RNS and GS formalisms is obtained, it is a good measure to check results against the established ones [9] [10]. As far as scattering amplitudes are concerned, these checks have already successfully been done at tree-level [11], one-loop [12] and two-loops [13].

Although the scattering amplitudes were shown to be equivalent, there is a huge difference in the amount of work required to obtain them, most notably in the two-loop case. The RNS two-loop computations of [10] span hundreds of pages because of complications related to the lack of manifest space-time supersymmetry, whereas it was obtained rather quickly in a seven-pages-long paper by Berkovits [14]. Furthermore, the computation of 114 is manifestly supersymmetric and therefore contains the result for all possible combination of external states related by supersymmetry, in deep contrast with the bosonic-only computation of [10].

One of the features of the pure spinor formalism that makes this simplification possible is the pure spinor superspace nature of its kinematic factors. In this paper we will explore this property and show how careful manipulations in pure spinor superspace can provide even further simplification of superstring scattering amplitude results. The upshot is that the one- and two-loop kinematic factors are completely determined (for Neveu-Schwarz and Ramond external states) once the tree-level amplitude is evaluated.

The massless four-point kinematic factors for the one- and two-loop amplitudes are given by [3] 12] 13]

$$
K_{1}=\left\langle\left(\lambda A^{1}\right)\left(\lambda \gamma^{m} W^{2}\right)\left(\lambda \gamma^{n} W^{3}\right) \mathcal{F}_{m n}^{4}\right\rangle+\operatorname{cycl} .(234),
$$




$$
K_{2}=\left\langle\left(\lambda \gamma^{m n p q r} \lambda\right) \mathcal{F}_{m n}^{1} \mathcal{F}_{p q}^{2} \mathcal{F}_{r s}^{3}\left(\lambda \gamma^{s} W^{4}\right)\right\rangle \Delta(1,3) \Delta(2,4)+\text { perm. }(1234),
$$

where $\Delta(i, j) \equiv \Delta\left(z_{i}, z_{j}\right)$ is the basic biholomorphic 1-form defined in [10]. Interestingly, the analogous expression for the tree-level amplitude has never been found, even after the explicit computations of [15]. So in section 2 we compute the massless four-point amplitude at tree-level to get

$$
K_{0}=\frac{1}{2} k_{1}^{m} k_{2}^{n}\left\langle\left(\lambda A^{1}\right)\left(\lambda A^{2}\right)\left(\lambda A^{3}\right) \mathcal{F}_{m n}^{4}\right\rangle-\left(k^{1} \cdot k^{3}\right)\left\langle A_{n}^{1}\left(\lambda A^{2}\right)\left(\lambda A^{3}\right)\left(\lambda \gamma^{n} W^{4}\right)\right\rangle+(1 \leftrightarrow 2) .
$$

Then we proceed to show in sections 3 and 4 that $K_{0}, K_{1}$ and $K_{2}$ satisfy the following identities

$$
\begin{gathered}
K_{0}=-\left\langle\left(\lambda A^{1}\right)\left(\lambda \gamma^{m} W^{2}\right)\left(\lambda \gamma^{n} W^{3}\right) \mathcal{F}_{m n}^{4}\right\rangle=-\frac{1}{3} K_{1} \\
K_{2}=-32 K_{0}[(u-t) \Delta(1,2) \Delta(3,4)+(s-t) \Delta(1,3) \Delta(2,4)+(s-u) \Delta(1,4) \Delta(2,3)]
\end{gathered}
$$

Finally, in section 5 we explain why some fermionic results reported in [16] contradict the above identities by pointing out a mistake made in [16] which invalidates its conclusions. After clarifying this issue, we compute the tree-level kinematic factor (1.3) for NeveuSchwarz and Ramond external states, which by the identities (1.4) and (1.5) also completely determine the one- and two-loop kinematic factors simultaneously.

\section{Massless four-point amplitude at tree-level}

Although the massless four-point amplitude at tree-level was already explicitly computed in [15], their derivation overlooked some identities in pure spinor superspace and hid the simplicity of the result. So in this section we compute the closed massless four-point amplitude at tree-level and extract the pure spinor superspace expression for its kinematic factor.

There are at least two motivations to pursue this goal. One is to neatly summarize the whole tree-level computation in one pure spinor superspace expression, which can later be used to find a relation with the one-loop amplitude, as will be done in section 3 . The other motivation is related to the ectoplasm method of [17], in which pure spinor superspace expressions in flat space can be used to find supersymmetric invariants in curved space backgrounds.

Following the tree-level prescription of [2] [4], the amplitude to compute is

$$
\mathcal{A}=\left\langle V^{1}\left(z_{1}, \bar{z}_{1}\right) V^{2}\left(z_{2}, \bar{z}_{2}\right) V^{3}\left(z_{3}, \bar{z}_{3}\right) \int_{C} d^{2} z_{4} U\left(z_{4}, \bar{z}_{4}\right)\right\rangle
$$


The closed string vertices are given by the holomorphic square of the open string vertices, $V(z, \bar{z})=\mathrm{e}^{i k \cdot X} \lambda^{\alpha} \bar{\lambda}^{\beta} A_{\alpha}(\theta) \bar{A}_{\beta}(\theta)$ and $U(z, \bar{z})=\mathrm{e}^{i k \cdot X} U(z) \bar{U}(\bar{z})$, where [3],

$$
U(z)=\partial \theta^{\alpha} A_{\alpha}(\theta)+A_{m}(\theta) \Pi^{m}+d_{\alpha} W^{\alpha}(\theta)+\frac{1}{2} N_{m n} \mathcal{F}^{m n}(\theta) .
$$

We note that standard $\mathrm{SL}(2, \mathrm{C})$ invariance allows us to fix $z_{1}=0, z_{2}=1$ and $z_{3}=\infty$, so $\left\langle\prod_{i=1}^{4}: \mathrm{e}^{i k^{i} \cdot X\left(z_{i}, \bar{z}_{i}\right)}:\right\rangle=\left|z_{4}\right|^{-\frac{1}{2} \alpha^{\prime} t}\left|1-z_{4}\right|^{-\frac{1}{2} \alpha^{\prime} u} \equiv M\left(z_{4}, \bar{z}_{4}\right)$. The first term of (2.2) does not contribute, while the second gives 2

$$
\left\langle A_{m}^{4} \Pi^{m}\left(z_{4}\right) \prod_{j=1}^{4}: \mathrm{e}^{i k^{j} \cdot X\left(z_{j}, \bar{z}_{j}\right)}:\right\rangle=\sum_{j=1}^{3} \frac{\alpha^{\prime}}{2} \frac{i k_{j}^{m}}{z_{j}-z_{4}}\left\langle\left(\lambda A^{1}\right)\left(\lambda A^{2}\right)\left(\lambda A^{3}\right) A_{m}^{4}\right\rangle M\left(z_{4}, \bar{z}_{4}\right) .
$$

Using the standard OPE's [3]

$$
N^{m n}\left(z_{4}\right) \lambda^{\alpha}\left(z_{j}\right)=\frac{\alpha^{\prime}}{4} \frac{\left(\lambda \gamma^{m n}\right)^{\alpha}}{z_{j}-z_{4}}, \quad d_{\alpha}\left(z_{4}\right) V\left(z_{j}\right)=-\frac{\alpha^{\prime}}{2} \frac{D_{\alpha} V}{z_{j}-z_{4}}
$$

we obtain the following OPE identity:

$$
\begin{gathered}
\left\langle\left(\lambda A^{1}\right)\left(\lambda A^{2}\right)\left(\lambda A^{3}\right)\left(d_{\alpha}\left(z_{4}\right) W_{4}^{\alpha}+\frac{1}{2} N^{m n}\left(z_{4}\right) \mathcal{F}_{m n}^{4}\right)\right\rangle= \\
=\frac{\alpha^{\prime}}{2\left(z_{1}-z_{4}\right)}\left\langle A_{m}^{1}\left(\lambda A^{2}\right)\left(\lambda A^{3}\right)\left(\lambda \gamma^{m} W^{4}\right)\right\rangle-(1 \leftrightarrow 2)+(1 \leftrightarrow 3) .
\end{gathered}
$$

To show this, one uses (2.4) to get

$$
\begin{gathered}
\left\langle\left(\lambda A^{1}\right)\left(z_{1}\right)\left(\lambda A^{2}\right)\left(z_{2}\right)\left(\lambda A^{3}\right)\left(z_{3}\right) d_{\alpha}\left(z_{4}\right) W_{4}^{\alpha}\right\rangle= \\
\frac{\alpha^{\prime}}{2\left(z_{1}-z_{4}\right)}\left\langle D_{\alpha}\left(\lambda A^{1}\right)\left(\lambda A^{2}\right)\left(\lambda A^{3}\right) W_{4}^{\alpha}\right\rangle-(1 \leftrightarrow 2)+(1 \leftrightarrow 3) .
\end{gathered}
$$

Concentrating for simplicity on the first term, the use of the super-Yang-Mills identity $D_{\alpha}(\lambda A)=-(\lambda D) A_{\alpha}+\left(\lambda \gamma^{m}\right)_{\alpha} A_{m}$ allows the numerator to be rewritten as

$$
\left\langle D_{\alpha}\left(\lambda A^{1}\right)\left(\lambda A^{2}\right)\left(\lambda A^{3}\right) W_{4}^{\alpha}\right\rangle=-\left\langle\left(\lambda D A_{\alpha}^{1}\right)\left(\lambda A^{2}\right)\left(\lambda A^{3}\right) W_{4}^{\alpha}\right\rangle+\left\langle A_{m}^{1}\left(\lambda A^{2}\right)\left(\lambda A^{3}\right)\left(\lambda \gamma^{m} W^{4}\right)\right\rangle
$$

As BRST-exact terms decouple, the first term in the right hand side of (2.6) becomes

$$
-\frac{\alpha^{\prime}}{2\left(z_{1}-z_{4}\right)}\left\langle\left(\lambda D A_{\alpha}^{1}\right)\left(\lambda A^{2}\right)\left(\lambda A^{3}\right) W_{4}^{\alpha}\right\rangle=-\frac{\alpha^{\prime}}{2\left(z_{1}-z_{4}\right)}\left\langle A_{\alpha}^{1}\left(\lambda A^{2}\right)\left(\lambda A^{3}\right)(\lambda D) W_{4}^{\alpha}\right\rangle
$$

2 To avoid the pollution of notation, we mostly omit the $z_{i}$ dependence in the superfields even when computing OPE's, as it does not prevent the proper understanding of the formulæ. 


$$
=-\frac{\alpha^{\prime}}{8\left(z_{1}-z_{4}\right)}\left\langle\left(\lambda \gamma^{m n} A^{1}\right)\left(\lambda A^{2}\right)\left(\lambda A^{3}\right) \mathcal{F}_{m n}^{4}\right\rangle
$$

However, this term is exactly canceled by the $\left(z_{1}-z_{4}\right)^{-1}$ contribution from the OPE

$$
\frac{1}{2}\left\langle\left(\lambda A^{1}\right)\left(\lambda A^{2}\right)\left(\lambda A^{3}\right)\left(N^{m n} \mathcal{F}_{m n}^{4}\right)\right\rangle=\frac{\alpha^{\prime}}{8\left(z_{1}-z_{4}\right)}\left\langle\left(\lambda \gamma^{m n} A^{1}\right)\left(\lambda A^{2}\right)\left(\lambda A^{3}\right) \mathcal{F}_{m n}^{4}\right\rangle+\ldots
$$

which finishes the proof of (2.5).

With the results (2.3) and (2.5), the correlation in the amplitude (2.1) reduces to

$$
\mathcal{A}=\left(\frac{\alpha^{\prime}}{2}\right)^{2} \int_{C} d^{2} z_{4}\left(\frac{F_{12}}{z_{4}}+\frac{F_{21}}{1-z_{4}}\right)\left(\frac{\bar{F}_{12}}{\bar{z}_{4}}+\frac{\bar{F}_{21}}{1-\bar{z}_{4}}\right)\left|z_{4}\right|^{-\frac{1}{2} \alpha^{\prime} t}\left|1-z_{4}\right|^{-\frac{1}{2} \alpha^{\prime} u},
$$

where $F_{12}=i k_{1}^{m}\left\langle\left(\lambda A^{1}\right)\left(\lambda A^{2}\right)\left(\lambda A^{3}\right) A_{m}^{4}\right\rangle+\left\langle A_{m}^{1}\left(\lambda A^{2}\right)\left(\lambda A^{3}\right)\left(\lambda \gamma^{m} W^{4}\right)\right\rangle$ and $F_{21}$ is obtained by exchanging $1 \leftrightarrow 2$. The integral can be evaluated using the following formula 18

$$
\int_{C} d^{2} z z^{N}(1-z)^{M} \bar{z}^{\bar{N}}(1-\bar{z})^{\bar{M}}=2 \pi \frac{\Gamma(1+N) \Gamma(1+M)}{\Gamma(2+N+M)} \frac{\Gamma(-1-\bar{N}-\bar{M})}{\Gamma(-\bar{N}) \Gamma(-\bar{M})} .
$$

After a few manipulations one finally gets

$$
\mathcal{A}=-2 \pi\left(\frac{\alpha^{\prime}}{2}\right)^{4} K_{0} \bar{K}_{0} \frac{\Gamma\left(-\frac{\alpha^{\prime} t}{4}\right) \Gamma\left(-\frac{\alpha^{\prime} u}{4}\right) \Gamma\left(-\frac{\alpha^{\prime} s}{4}\right)}{\Gamma\left(1+\frac{\alpha^{\prime} t}{4}\right) \Gamma\left(1+\frac{\alpha^{\prime} u}{4}\right) \Gamma\left(1+\frac{\alpha^{\prime} s}{4}\right)},
$$

where $K_{0}=\frac{1}{2}\left(u F_{12}+t F_{21}\right)$ is given by

$$
K_{0}=\left\langle\left(\partial_{m} A_{n}^{1}\right)\left(\lambda A^{2}\right) \partial^{m}\left(\lambda A^{3}\right)\left(\lambda \gamma^{n} W^{4}\right)\right\rangle-\frac{1}{2}\left\langle\partial^{m}\left(\lambda A^{1}\right) \partial^{n}\left(\lambda A^{2}\right)\left(\lambda A^{3}\right) \mathcal{F}_{m n}^{4}\right\rangle+(1 \leftrightarrow 2)
$$

which is the sought-for kinematic factor in pure spinor superspace. As will become clear later, it is convenient to rewrite (2.7) without explicit labels,

$$
K_{0}=2\left\langle\left(\partial_{m} A_{n}\right)(\lambda A) \partial^{m}(\lambda A)\left(\lambda \gamma^{n} W\right)\right\rangle-\left\langle(\lambda A) \partial^{m}(\lambda A) \partial^{n}(\lambda A) \mathcal{F}_{m n}\right\rangle .
$$

Furthermore, using the identities of [19] we will compute in section 5 the whole component expression of (2.7) (for Neveu-Schwarz and Ramond external states). We will see that expression (2.7) neatly summarizes the rather lenghty computations of [15]. 


\section{Relating tree-level and one-loop kinematic factors}

Using the well-known superfield equations of motion in the formulation of tendimensional Super-Yang-Mills theory in superspace [20] [21], one can show that

$$
Q \mathcal{F}_{m n}=2 \partial_{[m}\left(\lambda \gamma_{n]} W\right), \quad Q W^{\alpha}=\frac{1}{4}\left(\lambda \gamma^{m n}\right)^{\alpha} \mathcal{F}_{m n}, \quad Q A_{m}=\left(\lambda \gamma_{m} W\right)+\partial_{m}(\lambda A),
$$

where $Q=\oint \lambda^{\alpha} d_{\alpha}$ is the pure spinor BRST operator ${ }^{3}$. With these relations in hand we will show that (1.4) holds true. To prove this we note that $\left\langle(\lambda A) \partial^{m}(\lambda A)\left(Q A^{n}\right) F_{m n}\right\rangle=$ $-\left\langle(\lambda A) \partial^{m}(\lambda A) A^{n}\left(Q F_{m n}\right)\right\rangle$, which upon use of (3.1) and momentum conservation becomes

$$
\begin{gathered}
\left\langle(\lambda A) \partial^{m}(\lambda A)\left(Q A^{n}\right) F_{m n}\right\rangle=\left\langle(\lambda A) \partial^{m}(\lambda A) \partial_{m} A_{n}\left(\lambda \gamma^{n} W\right)\right\rangle \\
-\left\langle\partial_{n}(\lambda A) \partial_{m}(\lambda A) A^{n}\left(\lambda \gamma^{m} W\right)\right\rangle-\left\langle(\lambda A) \partial_{n} \partial_{m}(\lambda A) A^{n}\left(\lambda \gamma^{m} W\right)\right\rangle .
\end{gathered}
$$

The second term can be rewritten like

$$
\left\langle\partial_{n}(\lambda A) \partial_{m}(\lambda A) A^{n}\left(\lambda \gamma^{m} W\right)\right\rangle=-\left\langle(\lambda A)\left(\lambda \gamma^{m} W\right)\left[A^{n} \partial_{m} \partial_{n}(\lambda A)+\partial^{n}(\lambda A) \partial_{m} A_{n}\right]\right\rangle
$$

as can be shown by integrating $\partial^{m}$ by parts and using the equation of motion for $W^{\alpha}$. So,

$$
\left\langle(\lambda A) \partial^{m}(\lambda A)\left(Q A^{n}\right) F_{m n}\right\rangle=\left\langle(\lambda A) \partial^{m}(\lambda A)\left(\lambda \gamma^{n} W\right) F_{m n}\right\rangle-2\left\langle(\lambda A) \partial_{n} \partial_{m}(\lambda A) A^{n}\left(\lambda \gamma^{m} W\right)\right\rangle
$$

which implies that $\left\langle(\lambda A) \partial^{m}(\lambda A) \partial^{n}(\lambda A) F_{m n}\right\rangle=-2\left\langle(\lambda A) \partial_{n} \partial_{m}(\lambda A) A^{n}\left(\lambda \gamma^{m} W\right)\right\rangle$, or equivalently,

$$
\left\langle(\lambda A) \partial^{m}(\lambda A) \partial^{n}(\lambda A) F_{m n}\right\rangle=-2\left\langle(\lambda A) \partial_{n}\left(Q A_{m}\right) A^{n}\left(\lambda \gamma^{m} W\right)\right\rangle .
$$

Using $\left[Q, \partial^{n}\right]=0$ and the decoupling of BRST-trivial operators, equation (3.3) becomes

$$
\begin{gathered}
\left\langle(\lambda A) \partial^{m}(\lambda A) \partial^{n}(\lambda A) F_{m n}\right\rangle=2\left\langle(\lambda A)\left(\partial_{n} A_{m}\right)\left(Q A^{n}\right)\left(\lambda \gamma^{m} W\right)\right\rangle \\
=\left\langle(\lambda A)\left(\lambda \gamma^{m} W\right)\left(\lambda \gamma^{n} W\right) F_{m n}\right\rangle+2\left\langle\left(\partial_{n} A_{m}\right)(\lambda A) \partial^{n}(\lambda A)\left(\lambda \gamma^{m} W\right)\right\rangle .
\end{gathered}
$$

Plugging (3.4) in the tree-level kinematic factor (2.8) we finally obtain

$$
K_{0}=-\left\langle(\lambda A)\left(\lambda \gamma^{m} W\right)\left(\lambda \gamma^{n} W\right) F_{m n}\right\rangle=-\frac{1}{3} K_{1},
$$

which finishest the proof of (1.4).

3 We refer the reader to the lectures notes in the pure spinor formalism [21] for the basic definitions.

4 This proof was completed a few days after being told that Paul Howe had independently shown the same thing [22]. 


\section{Relating one- and two-loop kinematic factors}

To obtain a relation between the one- and two-loop kinematic factors we first need to show that $\left\langle\left(\lambda A^{1}\right)\left(\lambda \gamma^{m} W^{2}\right)\left(\lambda \gamma^{n} W^{3}\right) \mathcal{F}_{m n}^{4}\right\rangle$ is completely symmetric in the labels (1234). This can be done by noting that

$$
\left\langle\left(\lambda \gamma^{m n p q r} \lambda\right)\left(\lambda A^{1}\right)\left(W^{2} \gamma_{p q r} W^{3}\right) \mathcal{F}_{m n}^{4}\right\rangle=4\left\langle\left(\lambda A^{1}\right) Q\left[\left(W^{2} \gamma_{p q r} W^{3}\right)\right]\left(\lambda \gamma^{p q r} W^{4}\right)\right\rangle .
$$

Together with the identities $\left(\lambda \gamma^{m n} \gamma^{p q r} W^{2}\right)\left(\lambda \gamma_{p q r} W^{4}\right)=-48\left(\lambda \gamma^{[m} W^{2}\right)\left(\lambda \gamma^{n]} W^{4}\right)$ and $\left(\lambda \gamma^{m n p q r} \lambda\right)\left(W^{2} \gamma_{p q r} W^{3}\right)=-96\left(\lambda \gamma^{[m} W^{2}\right)\left(\lambda \gamma^{n]} W^{3}\right)$, equation (4.1) implies that

$$
\begin{gathered}
\left\langle\left(\lambda A^{1}\right)\left(\lambda \gamma^{m} W^{4}\right)\left(\lambda \gamma^{n} W^{2}\right) \mathcal{F}_{m n}^{3}\right\rangle+\left\langle\left(\lambda A^{1}\right)\left(\lambda \gamma^{m} W^{3}\right)\left(\lambda \gamma^{n} W^{4}\right) \mathcal{F}_{m n}^{2}\right\rangle= \\
=2\left\langle\left(\lambda A^{1}\right)\left(\lambda \gamma^{m} W^{2}\right)\left(\lambda \gamma^{n} W^{3}\right) \mathcal{F}_{m n}^{4}\right\rangle
\end{gathered}
$$

From (4.2) it follows that,

$$
K_{1-\text { loop }}=3\left\langle\left(\lambda A^{1}\right)\left(\lambda \gamma^{m} W^{2}\right)\left(\lambda \gamma^{n} W^{3}\right) \mathcal{F}_{m n}^{4}\right\rangle
$$

Furthermore, the independence of which vertex operator we choose to be non-integrated [1] implies total symmetry of $\left\langle\left(\lambda A^{1}\right)\left(\lambda \gamma^{m} W^{2}\right)\left(\lambda \gamma^{n} W^{3}\right) \mathcal{F}_{m n}^{4}\right\rangle$ in the labels (1234).

Now we can relate the one- and two-loop kinematic factors by noting that

$$
\begin{gathered}
\left(\lambda \gamma^{m n p q r} \lambda\right) \mathcal{F}_{m n}^{1} \mathcal{F}_{p q}^{2} \mathcal{F}_{r s}^{3}\left(\lambda \gamma^{s} W^{4}\right)=-4 Q\left[\left(\lambda \gamma^{r} \gamma^{m n} W^{2}\right)\left(\lambda \gamma^{s} W^{4}\right) \mathcal{F}_{m n}^{1} \mathcal{F}_{r s}^{3}\right] \\
-8 i k_{m}^{1}\left(\lambda \gamma_{n} W^{1}\right)\left(\lambda \gamma^{r} \gamma^{m n} W^{2}\right)\left(\lambda \gamma^{s} W^{4}\right) \mathcal{F}_{r s}^{3}
\end{gathered}
$$

where the pure spinor constraint $\left(\lambda \gamma^{m} \lambda\right)=0$ and the identity $\eta_{m n} \gamma_{\alpha(\beta}^{m} \gamma_{\gamma \delta)}^{n}=0$ must be used to show the vanishing of terms containing factors of $\left(\lambda \gamma^{m}\right)_{\alpha}\left(\lambda \gamma_{m}\right)_{\beta}$. Furthermore, as BRST-exact terms decouple from pure spinor correlations $\langle\ldots\rangle$, equation (4.4) implies

$$
\left\langle\left(\lambda \gamma^{m n p q r} \lambda\right) \mathcal{F}_{m n}^{1} \mathcal{F}_{p q}^{2} \mathcal{F}_{r s}^{3}\left(\lambda \gamma^{s} W^{4}\right)\right\rangle=+16 i k_{m}^{1}\left\langle\left(\lambda \gamma^{r} W^{1}\right)\left(\lambda \gamma^{m} W^{2}\right)\left(\lambda \gamma^{s} W^{4}\right) \mathcal{F}_{r s}^{3},\right\rangle
$$

where we have used $k_{m}^{1}\left(\lambda \gamma_{n} W^{1}\right)\left(\lambda \gamma^{r} \gamma^{m n} W^{2}\right)=-2 k_{m}^{1}\left(\lambda \gamma^{r} W^{1}\right)\left(\lambda \gamma^{m} W^{2}\right)$, which is valid when the equation of motion $k_{m}^{1}\left(\gamma^{m} W^{1}\right)_{\alpha}=0$ is satisfied.

Using $\left(\lambda \gamma_{m} W^{2}\right)=Q A_{m}^{2}-i k_{m}^{2}\left(\lambda A^{2}\right)$ and $\left\langle\left(\lambda \gamma^{r} W^{1}\right) Q\left(A_{2}^{m}\right)\left(\lambda \gamma^{s} W^{4}\right) \mathcal{F}_{r s}^{3}\right\rangle=0$ we arrive at the following pure spinor superspace identity

$$
\left\langle\left(\lambda \gamma^{m n p q r} \lambda\right) \mathcal{F}_{m n}^{1} \mathcal{F}_{p q}^{2} \mathcal{F}_{r s}^{3}\left(\lambda \gamma^{s} W^{4}\right)\right\rangle=-16\left(k^{1} \cdot k^{2}\right)\left\langle\left(\lambda A^{2}\right)\left(\lambda \gamma^{r} W^{1}\right)\left(\lambda \gamma^{s} W^{4}\right) \mathcal{F}_{r s}^{3}\right\rangle
$$

\footnotetext{
5 I thank Nathan Berkovits for suggesting (4.1) to me.
} 
Multiplying (4.6) by $\Delta(1,3) \Delta(2,4)$ and summing over permutations leads to the following identity,

$$
K_{2}=\frac{32}{3} K_{1}[(u-t) \Delta(1,2) \Delta(3,4)+(s-t) \Delta(1,3) \Delta(2,4)+(s-u) \Delta(1,4) \Delta(2,3)]
$$

where we used (4.3) and the standard Mandelstam variables $s=-2\left(k^{1} \cdot k^{2}\right), t=-2\left(k^{1} \cdot k^{4}\right)$, $u=-2\left(k^{2} \cdot k^{4}\right)$.

In view of the results in section 5, (4.7) not only provides a simple proof of two-loop equivalence with the (bosonic) RNS result of [10] but it also automatically implies the knowledge of the full amplitude, including fermionic external states.

\section{The complete tree-level, one- and two-loop kinematic factors}

The fermionic results reported in the first version of [16] are in direct contradiction with the identities (4.3) and (4.7). The two-loop kinematic factor (for 2F2B) was incorrectly argued to not have the simple form of (4.7) and the 2F2B one-loop computation of [16] does not obey identity (4.3). We clarify these issues by pointing out the mistake made in [16] which, strictly speaking, invalidates all its fermionic computations. After these issues are settled in the next paragraph, we compute the whole component expansion of (2.7), as that will automatically imply the full knowledge of $K_{2}$ for the first time. This is a remarkable example of the simplifying power of the pure spinor formalism.

In [16] the first component of $W^{\alpha}(\theta)$, denoted by $u^{\alpha}$, is considered to be bosonic instead of fermionic. So, in a strict sense, all fermionic computations in [16] are unreliable and all discussions based on symmetry properties of fermionic kinematic factors need review. In particular, the discussion of the $2 \mathrm{~F} 2 \mathrm{~B}$ kinematic factor at two-loops is wrong because we have proven in (4.7) that it is in fact proportional to the one-loop result.

This symmetry mistake in [16] is also apparent in its computation of $K_{1}^{2 \mathrm{~F} 2 \mathrm{~B}}$ at oneloop. One can check it in the first formula of section 3.3 , where the factor $\left(1-\pi_{34}\right)$ should be $\left(1+\pi_{34}\right)$. To see this note that the one-loop kinematic factor, with the cyclic permutations written out explicitly,

$$
\begin{gathered}
K_{1}=\left\langle\left(\lambda A^{1}\right)\left(\lambda \gamma^{m} W^{2}\right)\left(\lambda \gamma^{n} W^{3}\right) \mathcal{F}_{m n}^{4}\right\rangle+\left\langle\left(\lambda A^{1}\right)\left(\lambda \gamma^{m} W^{4}\right)\left(\lambda \gamma^{n} W^{2}\right) \mathcal{F}_{m n}^{3}\right\rangle+ \\
+\left\langle\left(\lambda A^{1}\right)\left(\lambda \gamma^{m} W^{3}\right)\left(\lambda \gamma^{n} W^{4}\right) \mathcal{F}_{m n}^{2}\right\rangle
\end{gathered}
$$


can be rewritten as

$$
\begin{gathered}
K_{1}=\left\langle\left(\lambda A^{1}\right)\left(\lambda \gamma^{m} W^{2}\right)\left(\lambda \gamma^{n} W^{3}\right) \mathcal{F}_{m n}^{4}\right\rangle+\left\langle\left(\lambda A^{1}\right)\left(\lambda \gamma^{m} W^{2}\right)\left(\lambda \gamma^{n} W^{4}\right) \mathcal{F}_{m n}^{3}\right\rangle+ \\
+\left\langle\left(\lambda A^{1}\right)\left(\lambda \gamma^{m} W^{3}\right)\left(\lambda \gamma^{n} W^{4}\right) \mathcal{F}_{m n}^{2}\right\rangle
\end{gathered}
$$

because $\left(\lambda \gamma^{m} W^{4}\right)\left(\lambda \gamma^{n} W^{2}\right)=-\left(\lambda \gamma^{n} W^{2}\right)\left(\lambda \gamma^{m} W^{4}\right)$ due to the fermionic nature of $W^{\alpha}(\theta)$. So the first line of equation (5.2) can be written in terms of the permutation symbol $\pi_{i j}$ as $\left(1+\pi_{34}\right)\left\langle\left(\lambda A^{1}\right)\left(\lambda \gamma^{m} W^{2}\right)\left(\lambda \gamma^{n} W^{3}\right) \mathcal{F}_{m n}^{4}\right\rangle$, and not with $\left(1-\pi_{34}\right)$ like shown 6 in [16]. Because of this mistake, the conclusion reached in [16] was that the first line of (5.1) vanished instead of being $+2\left\langle\left(\lambda A^{1}\right)\left(\lambda \gamma^{m} W^{3}\right)\left(\lambda \gamma^{n} W^{4}\right) \mathcal{F}_{m n}^{2}\right\rangle$, as one would conclude by using the identities proven in section 4. In fact, we know that all three terms in (5.1) are equal because of the total symmetry property demonstrated in this paper. So if one subtracts two of them the answer must be zero. The fact that in [16] the author concludes that $\left(1-\pi_{34}\right)\left\langle\left(\lambda A^{1}\right)\left(\lambda \gamma^{m} W^{2}\right)\left(\lambda \gamma^{n} W^{3}\right) \mathcal{F}_{m n}^{4}\right\rangle=0$ indicates that its computer codes are indeed correct.

In the sequence we use the following $\mathcal{N}=1$ super-Yang-Mills $\theta$ expansions

$$
\begin{gathered}
A_{\alpha}(x, \theta)=\frac{1}{2} a_{m}\left(\gamma^{m} \theta\right)_{\alpha}-\frac{1}{3}\left(\xi \gamma_{m} \theta\right)\left(\gamma^{m} \theta\right)_{\alpha}-\frac{1}{32} F_{m n}\left(\gamma_{p} \theta\right)_{\alpha}\left(\theta \gamma^{m n p} \theta\right) \\
+\frac{1}{60}\left(\gamma_{m} \theta\right)_{\alpha}\left(\theta \gamma^{m n p} \theta\right)\left(\partial_{n} \xi \gamma_{p} \theta\right)+\ldots \\
A_{m}(x, \theta)=a_{m}-\left(\xi \gamma_{m} \theta\right)-\frac{1}{8}\left(\theta \gamma_{m} \gamma^{p q} \theta\right) F_{p q}+\frac{1}{12}\left(\theta \gamma_{m} \gamma^{p q} \theta\right)\left(\partial_{p} \xi \gamma_{q} \theta\right)+\ldots \\
W^{\alpha}(x, \theta)=\xi^{\alpha}-\frac{1}{4}\left(\gamma^{m n} \theta\right)^{\alpha} F_{m n}+\frac{1}{4}\left(\gamma^{m n} \theta\right)^{\alpha}\left(\partial_{m} \xi \gamma_{n} \theta\right)+\frac{1}{48}\left(\gamma^{m n} \theta\right)^{\alpha}\left(\theta \gamma_{n} \gamma^{p q} \theta\right) \partial_{m} F_{p q}+\ldots \\
\mathcal{F}_{m n}(x, \theta)=F_{m n}-2\left(\partial_{[m} \xi \gamma_{n]} \theta\right)+\frac{1}{4}\left(\theta \gamma_{[m} \gamma^{p q} \theta\right) \partial_{n]} F_{p q}+\ldots,
\end{gathered}
$$

and the pure spinor superspace identities in the appendix of [19]. Here $\xi^{\alpha}(x)=\chi^{\alpha} \mathrm{e}^{i k \cdot x}$ and $a_{m}(x)=e_{m} \mathrm{e}^{i k \cdot x}$ describe the gluino and gluon respectively, while $F_{m n}=2 \partial_{[m} a_{n]}$ is the gluon field-strength.

Now we compute the whole component expansion of

$$
K_{0}=\frac{1}{2} k_{1}^{m} k_{2}^{n}\left\langle\left(\lambda A^{1}\right)\left(\lambda A^{2}\right)\left(\lambda A^{3}\right) \mathcal{F}_{m n}^{4}\right\rangle-\left(k^{1} \cdot k^{3}\right)\left\langle A_{m}^{1}\left(\lambda A^{2}\right)\left(\lambda A^{3}\right)\left(\lambda \gamma^{m} W^{4}\right)\right\rangle+(1 \leftrightarrow 2) .
$$

6 We also note that it is not necessary to make distinctions between $W^{\text {(even) }}$ and $W^{\text {(odd) }}$ to obtain (5.2). One can choose which superfields contribute with fermions $\left(\chi^{\alpha}\right)$ or bosons $\left(e_{m}\right)$ after these performing these pure spinor superspace manipulations. 
The first term doesn't contribute in the computation of $K_{0}\left(f_{1} f_{2} f_{3} f_{4}\right) \equiv K_{0}^{4 \mathrm{~F}}$, while the second leads tol

$$
\begin{gathered}
K_{0}^{4 \mathrm{~F}}=-\frac{1}{9}\left(k^{1} \cdot k^{3}\right)\left\langle\left(\lambda \gamma^{a} \theta\right)\left(\lambda \gamma^{b} \theta\right)\left(\lambda \gamma^{c} \chi^{4}\right)\left(\chi^{3} \gamma^{b} \theta\right)\left(\theta \gamma^{c} \chi^{1}\right)\left(\chi^{2} \gamma^{a} \theta\right)\right\rangle+(1 \leftrightarrow 2), \\
=\frac{1}{5760}\left[\left(\chi^{1} \gamma^{m} \chi^{2}\right)\left(\chi^{3} \gamma_{m} \chi^{4}\right)\left[\left(k^{2} \cdot k^{3}\right)-\left(k^{1} \cdot k^{3}\right)\right]-\frac{1}{12}\left(k^{3} \cdot k^{4}\right)\left(\chi^{1} \gamma^{m n p} \chi^{2}\right)\left(\chi^{3} \gamma_{m n p} \chi^{4}\right)\right] .
\end{gathered}
$$

Using the following Fierz identity [24]

$$
\left(\chi^{1} \gamma^{m n p} \chi^{2}\right)\left(\chi^{3} \gamma_{m n p} \chi^{4}\right)=24\left(\chi^{1} \gamma^{m} \chi^{3}\right)\left(\chi^{2} \gamma_{m} \chi^{4}\right)-12\left(\chi^{1} \gamma^{m} \chi^{2}\right)\left(\chi^{3} \gamma_{m} \chi^{4}\right)
$$

we arrive at

$$
K_{0}^{4 \mathrm{~F}}=-\frac{1}{2880}\left[\left(k^{1} \cdot k^{3}\right)\left(\chi^{1} \gamma^{m} \chi^{2}\right)\left(\chi^{3} \gamma_{m} \chi^{4}\right)+\left(k^{3} \cdot k^{4}\right)\left(\chi^{1} \gamma^{m} \chi^{3}\right)\left(\chi^{2} \gamma_{m} \chi^{4}\right)\right]
$$

Both terms of (5.3) contribute in the $K_{0}^{2 \mathrm{~B} 2 \mathrm{~F}} \equiv K_{0}\left(f_{1} f_{2} b_{3} b_{4}\right)$ kinematic factor,

$$
\begin{gathered}
K_{0}^{2 \mathrm{~B} 2 \mathrm{~F}}=-\frac{1}{36} k_{1}^{m} k_{2}^{n} F_{m n}^{4} e_{p}^{3}\left\langle\left(\lambda \gamma^{t} \theta\right)\left(\lambda \gamma^{u} \theta\right)\left(\lambda \gamma^{p} \theta\right)\left(\theta \gamma_{t} \chi^{1}\right)\left(\chi^{2} \gamma_{u} \theta\right)\right\rangle \\
-\frac{1}{24}\left(k^{1} \cdot k^{3}\right) F_{m n}^{4} e_{p}^{3}\left\langle\left(\lambda \gamma^{t} \theta\right)\left(\lambda \gamma^{p} \theta\right)\left(\lambda \gamma^{q} \gamma^{m n} \theta\right)\left(\theta \gamma_{q} \chi^{1}\right)\left(\chi^{2} \gamma_{t} \theta\right)\right\rangle+(1 \leftrightarrow 2) \\
=\frac{1}{5760} F_{m n}^{4} e_{p}^{3}\left[k_{1}^{m} k_{2}^{n}\left(\chi^{1} \gamma^{p} \chi^{2}\right)+\frac{1}{2}\left(k^{1} \cdot k^{3}\right)\left(\chi^{1} \gamma^{m n} \gamma^{p} \chi^{2}\right)\right]+(1 \leftrightarrow 2)
\end{gathered}
$$

It is worth noticing that the explicit computation of $K_{0}^{2 \mathrm{~B} 2 \mathrm{~F}}$ becomes easier if we use the identity (3.5) with a convenient choice for the labels in the right hand side, namely $K_{0}=-\left\langle\left(\lambda A^{1}\right)\left(\lambda \gamma^{m} W^{3}\right)\left(\lambda \gamma^{n} W^{4}\right) \mathcal{F}_{m n}^{2}\right\rangle$, because now one can check that only one term contributes

$$
\begin{gathered}
K_{0}^{2 \mathrm{~B} 2 \mathrm{~F}}=\frac{1}{24}\left\langle\left(\lambda \gamma^{p} \theta\right)\left(\lambda \gamma^{[m \mid} \gamma^{r s} \theta\right)\left(\lambda \gamma^{\mid n]} \gamma^{t u} \theta\right)\left(\theta \gamma_{p} \chi^{1}\right)\left(\chi^{2} \gamma_{n} \theta\right)\right\rangle k_{m}^{2} F_{r s}^{3} F_{t u}^{4} \\
=\frac{1}{5760} F_{m n}^{3} F_{r s}^{4}\left[-i\left(\chi^{1} \gamma^{r} \chi^{2}\right) \eta^{s m} k_{2}^{n}+\frac{i}{2}\left(\chi^{1} \gamma^{m n r} \chi^{2}\right) k_{2}^{s}\right]+(3 \leftrightarrow 4) .
\end{gathered}
$$

One can verify that (5.5) and (5.6) are in fact equal and equivalent to the RNS result (see for example [25]). This equality can also be regarded as a check of identity (3.5), which

7 I acknowledge the use of the GAMMA package [23] in these computations. 
is reassuring. The computation of $K_{0}^{4 \mathrm{~B}}$ is straightforward (and can also be deduced from the one-loop result of [12]). One can in fact check that

$$
\begin{aligned}
& K_{0}^{4 \mathrm{~B}}=\frac{1}{5760}\left[-\frac{1}{2}\left(e^{1} \cdot e^{3}\right)\left(e^{2} \cdot e^{4}\right) t s-\frac{1}{2}\left(e^{1} \cdot e^{4}\right)\left(e^{2} \cdot e^{3}\right) u s-\frac{1}{2}\left(e^{1} \cdot e^{2}\right)\left(e^{3} \cdot e^{4}\right) t u\right. \\
& +\left(k^{4} \cdot e^{1}\right)\left(k^{2} \cdot e^{3}\right)\left(e^{2} \cdot e^{4}\right) s+\left(k^{3} \cdot e^{2}\right)\left(k^{1} \cdot e^{4}\right)\left(e^{1} \cdot e^{3}\right) s \\
& +\left(k^{3} \cdot e^{1}\right)\left(k^{2} \cdot e^{4}\right)\left(e^{2} \cdot e^{3}\right) s+\left(k^{4} \cdot e^{2}\right)\left(k^{1} \cdot e^{3}\right)\left(e^{1} \cdot e^{4}\right) s \\
& +\left(k^{1} \cdot e^{2}\right)\left(k^{3} \cdot e^{4}\right)\left(e^{1} \cdot e^{3}\right) t+\left(k^{4} \cdot e^{3}\right)\left(k^{2} \cdot e^{1}\right)\left(e^{2} \cdot e^{4}\right) t \\
& +\left(k^{4} \cdot e^{2}\right)\left(k^{3} \cdot e^{1}\right)\left(e^{3} \cdot e^{4}\right) t+\left(k^{1} \cdot e^{3}\right)\left(k^{2} \cdot e^{4}\right)\left(e^{1} \cdot e^{2}\right) t \\
& +\left(k^{2} \cdot e^{1}\right)\left(k^{3} \cdot e^{4}\right)\left(e^{2} \cdot e^{3}\right) u+\left(k^{4} \cdot e^{3}\right)\left(k^{1} \cdot e^{2}\right)\left(e^{1} \cdot e^{4}\right) u \\
& \left.+\left(k^{4} \cdot e^{1}\right)\left(k^{3} \cdot e^{2}\right)\left(e^{3} \cdot e^{4}\right) u+\left(k^{2} \cdot e^{3}\right)\left(k^{1} \cdot e^{4}\right)\left(e^{1} \cdot e^{2}\right) u\right] \\
& =\frac{1}{2880} t_{8}^{m_{1} n_{1} m_{2} n_{2} m_{3} n_{3} m_{4} n_{4}} F_{m_{1} n_{1}}^{1} F_{m_{2} n_{2}}^{2} F_{m_{3} n_{3}}^{3} F_{m_{4} n_{4}}^{4},
\end{aligned}
$$

where we used the $t_{8}$ tensor definition of [26] [27].

Acknowledgements: I would like to thank UCLA and especially Eric D'Hoker for the hospitality I enjoyed during the completion of this work. I thank Nathan Berkovits for his faster-than-light email help when away and for his office door being always open when at the institute. I thank Christian Stahn for discussions regarding [16]. I also acknowledge FAPESP grant 04/13290-8 for financial support. 


\section{References}

[1] D. J. Gross and E. Witten, "Superstring Modifications Of Einstein's Equations," Nucl. Phys. B 277, 1 (1986).

[2] N. Berkovits, "Super-Poincare covariant quantization of the superstring," JHEP 0004, 018 (2000) arXiv:hep-th/0001035.

[3] N. Berkovits, "Multiloop amplitudes and vanishing theorems using the pure spinor formalism for the superstring," JHEP 0409, 047 (2004) [arXiv:hep-th/0406055].

[4] N. Berkovits, "Pure spinor formalism as an $\mathrm{N}=2$ topological string," JHEP 0510, 089 (2005) arXiv:hep-th/0509120.

[5] N. Berkovits and N. Nekrasov, "Multiloop superstring amplitudes from non-minimal pure spinor formalism," JHEP 0612, 029 (2006) [arXiv:hep-th/0609012.

[6] N. Berkovits, "Explaining the Pure Spinor Formalism for the Superstring," [hep-th] arXiv:0712.0324.

[7] N. Berkovits and D. Z. Marchioro, "Relating the Green-Schwarz and pure spinor formalisms for the superstring," JHEP 0501, 018 (2005) arXiv:hep-th/0412198.

[8] Y. Aisaka and Y. Kazama, "Origin of pure spinor superstring," JHEP 0505, 046 (2005) arXiv:hep-th/0502208.

[9] J. H. Schwarz, "Superstring Theory," Phys. Rept. 89, 223 (1982).

[10] E. D'Hoker and D. H. Phong, Nucl. Phys. B 715, 3 (2005) arXiv:hep-th/0501197

E. D'Hoker and D. H. Phong, Function," Nucl. Phys. B 715, 91 (2005) arXiv:hepth/0501196. E. D'Hoker and D. H. Phong, Nucl. Phys. B 639, 129 (2002) arXiv:hepth/0111040. E. D'Hoker and D. H. Phong, Nucl. Phys. B 636, 61 (2002) arXiv:hepth/0111016. E. D'Hoker and D. H. Phong, Nucl. Phys. B 636, 3 (2002) arXiv:hepth/0110283. E. D'Hoker and D. H. Phong, Phys. Lett. B 529, 241 (2002) arXiv:hepth/0110247.

[11] N. Berkovits and B. C. Vallilo, "Consistency of super-Poincare covariant superstring tree amplitudes," JHEP 0007, 015 (2000) arXiv:hep-th/0004171.

[12] C. R. Mafra, "Four-point one-loop amplitude computation in the pure spinor formalism," JHEP 0601, 075 (2006) arXiv:hep-th/0512052.

[13] N. Berkovits and C. R. Mafra, "Equivalence of two-loop superstring amplitudes in the pure spinor and RNS formalisms," Phys. Rev. Lett. 96, 011602 (2006) arXiv:hepth/0509234.

[14] N. Berkovits, "Super-Poincare covariant two-loop superstring amplitudes," JHEP 0601, 005 (2006) arXiv:hep-th/0503197.

[15] G. Policastro and D. Tsimpis, "R**4, purified," Class. Quant. Grav. 23, 4753 (2006) arXiv:hep-th/0603165.

[16] C. Stahn, "Fermionic superstring loop amplitudes in the pure spinor formalism," JHEP 0705, 034 (2007) [arXiv:0704.0015 [hep-th]]. 
[17] N. Berkovits, "Explaining pure spinor superspace," arXiv:hep-th/0612021.

[18] E. D'Hoker, M. Gutperle and D. H. Phong, "Two-loop superstrings and S-duality," Nucl. Phys. B 722, 81 (2005) arXiv:hep-th/0503180.

[19] N. Berkovits and C. R. Mafra, "Some superstring amplitude computations with the non-minimal pure spinor formalism," JHEP 0611, 079 (2006) arXiv:hep-th/0607187.

[20] E. Witten, "Twistor - Like Transform In Ten-Dimensions," Nucl. Phys. B 266, 245 (1986).

[21] N. Berkovits, "ICTP lectures on covariant quantization of the superstring," arXiv:hepth/0209059.

[22] Paul Howe, private communication.

[23] U. Gran, "GAMMA: A Mathematica package for performing Gamma-matrix algebra and Fierz transformations in arbitrary dimensions," arXiv:hep-th/0105086.

[24] V. Alexandrov, D. Krotov, A. Losev and V. Lysov, "On Pure Spinor Superfield Formalism," JHEP 0710, 074 (2007) arXiv:0705.2191 [hep-th]].

[25] K. Lee and W. Siegel, "Simpler superstring scattering," JHEP 0606, 046 (2006) arXiv:hep-th/0603218.

[26] M. B. Green, J. H. Schwarz and E. Witten, "Superstring Theory. Vol. 1: Introduction," Cambridge, Uk: Univ. Pr. (1987) 469 P. ( Cambridge Monographs On Mathematical Physics)

[27] M. B. Green, J. H. Schwarz and E. Witten, "Superstring Theory. Vol. 2: Loop Amplitudes, Anomalies And Phenomenology," Cambridge, Uk: Univ. Pr. (1987) 596 P. ( Cambridge Monographs On Mathematical Physics) 